\title{
On Banach contraction principle in a cone metric space
}

\author{
Shobha Jain ${ }^{a}$, Shishir Jain ${ }^{b, *}$, Lal Bahadur Jain ${ }^{c}$ \\ ${ }^{a}$ Quantum School of Technology, Roorkee (U.K), India. \\ ${ }^{b}$ Shri Vaishnav Institute of Technology and Science, Indore (M.P.), India. \\ ${ }^{c}$ Retd. Principal, Govt. Arts and Commerce College Indore (M.P.), India. \\ This paper is dedicated to Professor Ljubomir Ćirić \\ Communicated by Professor V. Berinde
}

\begin{abstract}
The object of this paper is to establish a generalized form of Banach contraction principle for a cone metric space which is not necessarily normal. This happens to be a generalization of all different forms of Banach contraction Principle, which have been arrived at in L. G. Huang and X. Zhang [L. G. Huang and X. Zhang, J. Math. Anal. Appl 332 (2007), 1468-1476] and Sh. Rezapour, R. Hamlbarani [Sh. Rezapour, R. Hamlbarani, J. Math. Anal. Appl. 345 (2008) 719-724] and D. Ilic, V. Rakocevic [D. Ilic, V. Rakocevic, Applied Mathematics Letters 22 (2009), 728-731]. It also results that the theorem on quasi contraction of Ciric̃ [L. J. B. C̃iric̃, Proc. American Mathematical Society 45 (1974), 999-1006]. for a complete metric space also holds good in a complete cone metric space. All the results presented in this paper are new. (C)2012. All rights reserved.
\end{abstract}

Keywords: Cone metric space, common fixed points.

\section{Introduction}

There has been a number of generalizations of metric space. One such generalization is a cone metric space. In the second half of previous century a lot of work has been done in a K-metric space, which is in the setting of cone in a real normed linear space and variously defined notions of convergence and a Cauchy

\footnotetext{
${ }^{*}$ Corresponding author

Email addresses: shobajain1@yahoo.com ( Shobha Jain), jainshishir11@rediffmail.com (Shishir Jain), lalbahdurjain11@yahoo.com ( Lal Bahadur Jain)
} 
sequence [13]. However, another school in U.S.S.R [7, 8, 9, 10] worked in K- metric space in the setting of a Banach space $B$ and a closed cone in it in the name of a generalized metric space or a SKS metric space. Recently, in [3] Huang and Zhang defined cone metric space in the same setting of a real Banach space E ordered with a closed cone $\mathrm{P}$ in it with int $P \neq \Phi$ defining convergence and a Cauchy sequence with respect to interior points of $P$. In this space they replaced the set of real numbers of a metric space by an ordered Banach Space and gave some fundamental results for a self map satisfying a contractive condition assuming the normality of cone metric space.

Recently, Rezapour and Hamlbarani [1] omitted the assumption of normality in cone metric space, which is a milestone in developing fixed point theory in cone metric space.In [5], the authors introduced the concept of a compatible pair of self maps in a cone metric space and established a basic result for a non-normal cone metric space with an example, while in [6] weakly compatible maps have been studied. In this paper we are proving a common fixed point theorem for a sequence of self maps satisfying a generalized contractive condition for a non-normal cone metric space. It results in a generalized form of Banach contraction principle in this space.

\section{Preliminaries}

Definition 2.1. 3] Let $E$ be a real Banach space and $P$ be a subset of $E$. $P$ is called a cone if

(i) $P$ is a closed, nonempty and $P \neq\{0\}$;

(ii) $a, b \in R, a, b \geq 0, x, y \in P$ imply $a x+b y \in P$;

(iii) $x \in P$ and $-x \in P$ imply $x=0$.

Given a cone $P \subseteq E$, we define a partial ordering " $\leq$ " in $E$ by $x \leq y$ if $y-x \in P$. We write $x<y$ to denote $x \leq y$ but $x \neq y$ and $x<<y$ to denote $y-x \in P^{0}$, where $P^{0}$ stands for the interior of $P$.

$P$ is called normal if for some $M>0$ for $x, y \in E, 0 \leq x \leq y$ implies

$\|x\| \leq M \mid y \|$.

Proposition 2.2. Let $P$ be a cone in a real Banach space $E$. If for $a \in P$ and $a \leq k a$, for some $k \in[0,1)$ then $a=0$.

Proof: For $a \in P, k \in[0,1)$ and $a \leq k a$ gives $(k-1) a \in P$ implies $-(1-k) a \in P$. Therefore by (ii) we have $-a \in P$, as $1 /(1-k)>0$. Hence $a=0$, by $($ iii $)$.

Proposition 2.3. 4 Let $P$ be a cone is a real Banach space $E$ with non-empty interior If for $a \in E$ and $a<<c$, for all $c \in P^{0}$, then $a=0$.

Remark 2.4. [1] $\lambda P^{0} \subseteq P^{0}$, for $\lambda>0$ and $P^{0}+P^{0} \subseteq P^{0}$.

Definition 2.5. [3] Let $X$ be a nonempty set and $P$ be a cone in a real Banach space $E$. Suppose the mapping $d: X \times X \rightarrow E$ satisfies:

(a) $0 \leq d(x, y)$, for all $x, y \in X$ and $d(x, y)=0$, if and only if $x=y$;

(b) $d(x, y)=d(y, x)$, for all $x, y \in X$;

(c) $d(x, y) \leq d(x, z)+d(z, y)$, for all $x, y, z \in X$.

Then $d$ is called a cone metric on $X$, and $(X, d)$ is called a cone metric space. If $P$ is normal, then $(X, d)$ is said to be a a normal cone metric space.

Example 2.6. 3] Let $E=R^{2}, P=\{(x, y) \in E: x \geq 0, y \geq 0\}$ and $X=R$. For $x, y \in R$ define $d(x, y)=|x-y|(1, \alpha)$ where $\alpha \geq 0$ is some fixed constant. Then $(X, d)$ is a cone metric space.

Example 2.7. Let $E=C_{R}^{2}[0,1]$ with the norm $\|f\|=\|f\|_{\infty}+\left\|f^{\prime}\right\|_{\infty}$. Consider the cone $P=\{f \in E: f \geq$ $0\}$. Then $P$ is not a normal cone as shown in [11]. Taking $X=\{1,1 / 2,1 / 3 \ldots\}$ we define $d: X \times X \rightarrow P$ by $d\left(\frac{1}{m}, \frac{1}{n}\right)=f_{m n}$, where $f_{m n}(t)=\left|\frac{1}{m}-\frac{1}{n}\right| t$, for all $t \in[0,1]$. Then $(X, d)$ is a non-normal cone metric space. $(X, d)$ is not a metric space as it is not normal. 
Definition 2.8. 3] Let $(X, d)$ be a cone metric space with respect to a cone in a real Banach space $E$ with non-empty interior. Let $\left\{x_{n}\right\}$ be a sequence in $X$ and $x \in X$. If for every $c \in E$ with $0<<c$ there is a positive integer $N_{c}$ such that for all $n>N_{c}, d\left(x_{n}, x\right)<<c$, then the sequence $\left\{x_{n}\right\}$ is said to converges to $x$, and $x$ is called limit of $\left\{x_{n}\right\}$. We write $\lim _{n \rightarrow \infty} x_{n}=x$ or $x_{n} \rightarrow x$, as $n \rightarrow \infty$.

Definition 2.9. [3] Let $(X, d)$ be a cone metric space with respect to a cone with nonempty interior in a real Banach space $E$. Let $\left\{x_{n}\right\}$ be a sequence in $X$. If for any $c \in E$ with $0<<c$ there is a positive integer $N_{c}$ such that for all $n, m>N_{c}, d\left(x_{n}, x_{m}\right)<<c$, then the sequence $\left\{x_{n}\right\}$ is said to be a Cauchy sequence in $X$.

In the following $(X, d)$ will stand for a cone metric space with respect to a cone $P$ with $P^{0} \neq \phi$ in a real Banach space $E$ and $\leq$ is partial ordering in $E$ with respect to $P$

Remark 2.10. It follows from above definitions that if $\left\{x_{2 n}\right\}$ is a subsequence of a Cauchy sequence $\left\{x_{n}\right\}$ in a cone metric space $(X, d)$ and $x_{2 n} \rightarrow z$ then $x_{n} \rightarrow z$.

Definition 2.11. [3] Let $(X, d)$ be a cone metric space. If every Cauchy sequence in $X$ is convergent in $X$, then $X$ is called a complete cone metric space.

Proposition 2.12. Let $(X, d)$ be a cone metric space and $P$ be a cone in a real Banach space E. If $u \leq$ $v, v<<w$ then $u<<w$.

Lemma 2.13. Let $(X, d)$ be a cone metric space and $P$ be a cone in a real Banach space $E$ and $k_{1}, k_{2}, k>0$ are some fixed real numbers. If $x_{n} \rightarrow x, y_{n} \rightarrow y$ in $X$ and for some $a \in P$

(1.1) $k a \leq k_{1} d\left(x_{n}, x\right)+k_{2} d\left(y_{n}, y\right)$, for all $n>N$, for some integer $N$,

then $a=0$.

Proof As $x_{n} \rightarrow x$, and $y_{n} \rightarrow y$ for $c \in P^{0}$ there exists a positive integer $N_{c}$ such that $\frac{c}{\left(k_{1}+k_{2}\right)}-d\left(x_{n}, x\right), \frac{c}{\left(k_{1}+k_{2}\right)}-d\left(y_{n}, y\right) \in P^{0}$, for all $n>N_{c}$.

Therefore by Remark 2.4, we have

$\frac{k_{1} c}{\left(k_{1}+k_{2}\right)}-k_{1} d\left(x_{n}, x\right), \frac{k_{2} c}{\left(k_{1}+k_{2}\right)}-k_{2} d\left(y_{n}, y\right) \in P^{0}$, for all $n>N_{c}$.

Again by adding and Remark 2.4, we have

$c-k_{1} d\left(x_{n}, x\right)-k_{2} d\left(y_{n}, y\right) \in P^{0}$ for all $n>\max \left\{N, N_{c}\right\}$.

From (1.1) and Proposition 2.12 we have $k a<<c$, for each $c \in P^{0}$. By Proposition 2.3, we have $a=0$, as $k>0$.

\section{MAIN RESULTS}

Theorem 3.1. Let $(X, d)$ be a complete cone metric space with respect to a cone $P$ contained in a real Banach space E. Let $\left\{T_{n}\right\}$ be a sequence of self maps on $X$ satisfying:

(3.1.1) For some $\lambda, \mu, \delta, \alpha, \beta \in[0,1)$ with $\lambda+\mu+\delta+2 \alpha<1$, or else $\lambda+\mu+\delta+2 \beta<1$, for all $x, y \in X$ $d\left(T_{i} x, T_{j} y\right) \leq \lambda d\left(T_{i} x, x\right)+\mu d\left(T_{j} y, y\right)+\delta d(x, y)+\alpha d\left(x, T_{j} y\right)+\beta d\left(T_{i} x, y\right)$.

For $x_{0} \in X$, let $x_{n}=T_{n} x_{n-1}$, for all $n$. Then the sequence $\left\{x_{n}\right\}$ converges in $X$ and its limit $u$ is a common fixed point of all the maps of the sequence $\left\{T_{n}\right\}$. This fixed point is unique if $\delta+\alpha+\beta<1$.

Proof. We show that $\left\{x_{n}\right\}$ is a Cauchy sequence in $X$.

Step I: Taking $x=x_{n-1}, y=x_{n}$ and $i=n, j=n+1$ in (3.1.1) we get,

$$
\begin{aligned}
d\left(T_{n} x_{n-1}, T_{n+1} x_{n}\right) & \leq \lambda d\left(T_{n} x_{n-1}, x_{n-1}\right)+\mu d\left(T_{n+1} x_{n}, x_{n}\right)+\delta\left(x_{n-1}, x_{n}\right)+ \\
& \alpha d\left(x_{n-1}, T_{n+1} x_{n}\right)+\beta d\left(T_{n} x_{n-1}, x_{n}\right) .
\end{aligned}
$$

As $x_{n}=T_{n} x_{n-1}$, we have

$$
\begin{aligned}
d\left(x_{n}, x_{n+1}\right) & \leq \lambda d\left(x_{n}, x_{n-1}\right)+\mu d\left(x_{n+1}, x_{n}\right)+\delta\left(x_{n-1}, x_{n}\right)+\alpha d\left(x_{n-1}, x_{n+1}\right)+\beta d\left(x_{n}, x_{n}\right) \\
& \leq \lambda d\left(x_{n}, x_{n-1}\right)+\mu d\left(x_{n+1}, x_{n}\right)+\delta\left(x_{n-1}, x_{n}\right)+\alpha\left[d\left(x_{n-1}, x_{n}\right)+d\left(x_{n}, x_{n+1}\right)\right]
\end{aligned}
$$


Writing $d\left(x_{n}, x_{n+1}\right)=d_{n}$, we have

$d_{n} \leq \lambda d_{n-1}+\mu d_{n}+\delta d_{n-1}+\alpha\left[d_{n}+d_{n-1}\right]$,

i.e.

$(1-\mu-\alpha) d_{n}=(\lambda+\delta+\alpha) d_{n-1}$,

which implies

$$
d_{n} \leq h d_{n-1}
$$

if $h=\frac{(\lambda+\delta+\alpha)}{1-\mu-\alpha}$.

As $\lambda+\mu+\delta+2 \alpha<1$ we obtain that $h<1$.

Now

$d_{n} \leq h d_{n-1} \leq h^{2} d_{n-2} \leq h^{3} d_{n-3} \leq \ldots \leq h^{n} d_{0}$, where $d_{0}=d\left(x_{0}, x_{1}\right)$.

Also

$d\left(x_{n+p}, x_{n}\right) \leq d\left(x_{n+p}, x_{n+p-1}\right)+d\left(x_{n+p-1}, x_{n+p-2}\right)+\ldots+d\left(x_{n+1}, x_{n}\right)$,

i. e.

$$
\begin{aligned}
d\left(x_{n+p}, x_{n}\right) & \leq d_{n+p-1}+d_{n+p-2}+\cdots+d_{n} . \\
& =d_{n}+d_{n+1}+\ldots d_{n+p-1} \\
& =h^{n}\left[1+h+h^{2}+h^{3}+\ldots+h^{p-1}\right] d_{0} \\
& \leq h^{n} d_{0} /(1-h)
\end{aligned}
$$

as $h<1$ and $P$ is closed. Thus we obtain that

$$
d\left(x_{n+p}, x_{n}\right) \leq h^{n} d_{0} /(1-h) .
$$

Now for $c \in P^{0}$, there exists $r>0$ such that $c-y \in P^{0}$, if $\|y\|<r$. Choose a positive integer $N_{c}$ such that for all $n \geq N_{c},\left\|h^{n} d_{0} /(1-h)\right\|<r$, which implies $c-h^{n} d_{0} /(1-h) \in P^{0}$ and $h^{n} d_{0} /(1-h)-d\left(x_{n+p}, x_{n}\right) \in P$, using (3.2).

So we have $c-d\left(x_{n+p}, x_{n}\right) \in P^{0}$, for all $n>N_{c}$ and for all $p$, by Proposition 2.12. This implies $d\left(x_{n+p}, x_{n}\right)<<c$, for all $n>N_{c}$, for all $p$. Hence $\left\{x_{n}\right\}$ is a Cauchy sequence in $X$, which is complete.

Let $x_{n} \rightarrow u$.

Step II: For an arbitrary fixed $m$ we show that $T_{m} u=u$.

Now,

$$
\begin{aligned}
d\left(T_{m} u, u\right) & \leq d\left(T_{m} u, T_{n} x_{n-1}\right)+d\left(T_{n} x_{n-1}, u\right), \\
& =d\left(x_{n}, u\right)+d\left(T_{m} u, T_{n} x_{n-1}\right) .
\end{aligned}
$$

Using (3.1.1) with $x=x_{n-1}, y=u, i=n$ and $j=m$ we have

$$
\begin{aligned}
d\left(T_{m} u, u\right) \leq & d\left(x_{n}, u\right)+\lambda d\left(T_{n} x_{n-1}, x_{n-1}\right)+\mu d\left(T_{m} u, u\right) \\
& +\delta d\left(u, x_{n-1}\right)+\alpha d\left(T_{m} u, x_{n-1}\right)+\beta d\left(u, T_{n} x_{n-1}\right) \\
& =d\left(x_{n}, u\right)+\mu d\left(T_{m} u, u\right)+\lambda d\left(x_{n}, x_{n-1}\right) \\
& +\delta d\left(u, x_{n-1}\right)+\alpha d\left(T_{m} u, x_{n-1}\right)+\beta d\left(u, x_{n}\right), \\
& \leq d\left(x_{n}, u\right)+\mu d\left(T_{m} u, u\right)+\lambda\left[d\left(x_{n}, u\right)+d\left(u, x_{n-1}\right)\right] \\
& +\delta d\left(u, x_{n-1}\right)+\alpha\left[d\left(T_{m} u, u\right)+d\left(u, x_{n-1}\right)\right]+\beta\left[d\left(u, x_{n}\right) .\right.
\end{aligned}
$$

So

$[1-\mu-\alpha] d\left(T_{m} u, u\right) \leq[\mu+\delta+\alpha] d\left(x_{n-1}, u\right)+[1+\lambda+\beta] d\left(u, x_{n}\right)$.

As $\left\{x_{n}\right\} \rightarrow u,\left\{x_{n-1}\right\} \rightarrow u$, and $1-\mu-\alpha>0$, using Lemma 2.13, we have $d\left(T_{m} u, u\right)=0$, and we get $T_{m} u=u$. Thus $u$ is a common fixed point of all the maps of the sequence $\left\{T_{n}\right\}$.

Step III (Uniqueness): Let $T_{n} z=z$, for all $n$, be another common fixed point of all the maps of the sequence $\left\{T_{n}\right\}$. Now

$d(z, u)=d\left(T_{n} z, T_{n} u\right)$.

Taking $x=z$ and $y=u$ with $i=j=n$ in (3.1.1) we get $d(z, u) \leq \lambda d\left(T_{n} z, z\right)+\mu d\left(T_{n} u, u\right)+\delta d(z, u)+\alpha d\left(z, T_{n} u\right)+\beta d\left(T_{n} z, u\right)$,

which gives

$d(z, u) \leq(\delta+\alpha+\beta) d(z, u)$. 
As $\delta+\alpha+\beta<1$, using Proposition 2.2, we have $d(z, u)=0$ i. e. $u=z$. Thus $\mathrm{u}$ is the unique common fixed point of all the maps of the sequence $\left\{T_{n}\right\}$. To see the sufficiency of the alternate condition $\lambda+\mu+\delta+2 \beta<1$, in step I we choose $x=u, y=x_{n-1}$ with $i=n+1$ and $j=n$ in (3.1.1) to obtain $(1-\lambda-\beta) d_{n} \leq(\mu+\delta+\beta) d_{n-1}$. Thus $d_{n} \leq h^{\prime} d_{n-1}$, where $h^{\prime}=\frac{(\mu+\delta+\beta)}{1-\lambda-\beta}<1$.

Again in step II we choose $x=u, y=x_{n-1} i=m, j=n$ in (3.1.1) receiving $(1-\lambda-\beta) d\left(T_{m}(u), u\right) \leq \ldots$ and we get $T_{m} u=u, \forall m$.

Theorem 3.2. Let $(X, d)$ be a complete cone metric space with respect to a cone $P$ contained in a real Banach space $E$. Let $\left\{A_{n}\right\}$ be a sequence of self maps in $X$ satisfying:

(3.2.1) For some $\lambda, \mu, \delta, \alpha, \beta \in[0,1)$ with $\lambda+\mu+\delta+2 \alpha<1$, or else $\lambda+\mu+\delta+2 \beta<1$ and $\delta+\alpha+\beta<1$, there exists positive integer $m_{i}$, for each $i$, such that for all $x, y \in X$

$d\left(A_{i}^{m_{i}} x, A_{j}^{m_{j}} y\right) \leq \lambda d\left(A_{i}^{m_{i}} x, x\right)+\mu d\left(A_{j}^{m_{j}} y, y\right)+\delta(x, y)+\alpha d\left(x, A_{j}^{m_{j}} y\right)+\beta d\left(A_{i}^{m_{i}} x, y\right)$. Then all the maps of the sequence $\left\{A_{n}\right\}$ have a unique common fixed point in $X$.

Proof. In view of (3.2.1) and using Theorem 3.1 all the maps of the sequence $\left\{A_{i}^{m_{i}}\right\}$ have a unique common fixed point, say z. Hence $A_{i}^{m_{i}} z=z$, for all i. Now $A_{1}^{m_{1}} z=z$, implies $A_{1}^{m_{1}} A_{1} z=A_{1} z$. Taking $x=A_{1} z, y=$ $z, i=1$ and $j=2$ in (3.2.1) we have $A_{1} z=z$. Continuing in similar way it follows that $A_{i} z=z$, for all i.Thus $\mathrm{z}$ is a common fixed point of all the maps of the sequence $\left\{A_{i}\right\}$. Its uniqueness follows from the fact that $A_{i} z=z$, implies $A_{i}^{m_{i}} z=z$, for all i.

Example 3.3. (of Theorem 3.2 Let $X=[0,1], E=R^{2}, P=\left\{(x, y) \in R^{2}: x \geq 0, y \geq 0\right\} \subseteq R^{2}$, be a cone in $E$. Fix a real number $\gamma>0$. We define $d: X \times X \rightarrow E$ by $d(x, y)=|x-y|(1, \gamma)$. Then $(X, d)$ is a complete cone metric space. Define $\left\{A_{n}\right\}$ on $X$ as follows:

$A_{n}(x)= \begin{cases}0, & \text { if } x \in\left[0, \frac{1}{n+2}\right] \\ \frac{1}{n+3}, \text { otherwise. } & \end{cases}$

Taking $m_{i}=2$, for all $\mathrm{i}$. Then the maps $A_{1}^{2}, A_{2}^{2}, A_{3}^{2}, \ldots$ satisfy the condition (3.2.1) for $\lambda=\mu=\delta=\frac{1}{15}$ and $\alpha=\beta=\frac{1}{10}$. Hence by Theorem 3.2, all the maps of the sequence $\left\{A_{n}\right\}$ have a unique common fixed point $(u=0)$ in $\mathrm{X}$.

Taking $T_{1}=T_{2}=T_{3}=\cdots=T_{n-1}=T_{n}=\cdots=A$ in Theorem 3.1, we get the following general form of Banach contraction principal in a cone metric space which is not necessarily normal

Theorem 3.4. Let $(X, d)$ be a complete cone metric space with respect to a cone $P$ contained in a real Banach space $E$ and $A$ be a self map in $X$ satisfying:

(3.4.1) For some $\lambda, \mu, \delta, \alpha, \beta \in[0,1)$ with $\lambda+\mu+\delta+2 \alpha<1$, or else $\lambda+\mu+\delta+2 \beta<1$, for all $x, y \in X$ $d(A x, A y) \leq \lambda d(A x, x)+\mu d(A y, y)+\delta(x, y)+\alpha d(x, A y)+\beta d(A x, y)$.

Then for each $x$ in $X$ the sequence $\left\{A^{n} x\right\}$ converges in $X$ and its limit $u$ is a fixed point of A.This fixed point is unique if $\delta+\alpha+\beta<1$.

In [3] L. G. Huang, X. Zhang and in [11] Sh. Rezapour, R. Hamlbarani proved following various forms of Banach contraction Principle in a normal Cone metric space and in a cone metric space respectively :

Theorem 1[3] and Theorem 2.3[11] : Let $(X, d)$ be a complete cone metric space, Suppose the mapping $T: X \times X \rightarrow X$ satisfies the contractive condition $d(T x, T y) \leq k d(x, y)$, for all $x, y \in X$,

where $k \in[0,1)$ is a constant. Then $T$ has a unique fixed point in $X$. For each $x \in X$, the iterative sequence $\left\{T^{n} x\right\}$ converges to the fixed point.

Theorem 3[3] and Theorem 2.6 [11]: Let $(X, d)$ be a complete cone metric space. Suppose the mapping $T: X \times X \rightarrow X$ satisfies the contractive condition $d(T x, T y) \leq k[d(T x, x)+d(T y, y)]$ for all $x, y \in X$, where $k \in[0,1 / 2)$ is a constant. Then $T$ has a unique fixed point in $X$. And for $x \in X$, the iterative sequence $\left\{T^{n} x\right\}$ converges to the fixed point. 
Theorem $4[3]$ and Theorem 2.7 [11]: Let $(X, d)$ be a complete cone metric space. Suppose the mapping $T: X \times X \rightarrow X$ satisfies the contractive condition $d(T x, T y) \leq k[d(T x, y)+d(T y, x)]$ for all $x, y \in X$, where $k \in[0,1 / 2)$ is a constant. Then $T$ has a unique fixed point in $X$. For each $x \in X$, the iterative sequence $\left\{T^{n} x\right\}$ converges to the fixed point.

Theorem 2.8 [11]: Let $(X, d)$ be a complete cone metric space. Suppose the mapping $T: X \times X \rightarrow X$ satisfies the contractive condition $d(T x, T y) \leq k d(x, y)+l d(y, T x)$ for all $x, y \in X$, where $k, l \in[0,1)$ are constants. Then $T$ has a fixed point in $X$. Also the fixed point of $T$ is unique whenever $k+l<1$.

Remark 3.5. Above Theorems of [3] and [11] follow from Theorem 3.4 of this paper by taking :

(a) $\lambda=\mu=\alpha=\beta=0$ and $\delta=k$,

(b) $\lambda=\mu=k$ and $\delta=\alpha=\beta=0$,

(c) $\lambda=\mu=\delta=0$ and $\alpha=\beta=k$, and

(d) $\lambda=\mu=\alpha=0, \delta=k$, and $\beta=l$

respectively in it.

Precisely,Theorem 3.4 synthesizes and generalizes all the results of [3] and [11] for a non-normal cone metric space. Theorem 3.1 is a general form of Banach contraction principle in a complete cone metric space which is not necessarily normal.

Definition 3.6. [4] (Quasi contraction)A self-map $\mathrm{f}$ on a cone metric space $(X, d)$ is said to be a quasi contraction if for a fixed $\lambda \in(0,1), d(f x, f y) \leq \lambda u$ for every $x, y \in X$, where

$$
u \in\{d(x, y), d(x, f x), d(y, f y), d(x, f y), d(y, f x)\} .
$$

Theorem 2.1 [4]: Let $(X, d)$ be a complete cone metric space and $\mathrm{P}$ be a normal cone. Then a quasi contraction $\mathrm{f}$ has a unique fixed point in $X$ and for each $x \in X$ the iterative sequence $\left\{f^{n}(x)\right\}$ converges to the fixed point.

Remark 3.7. Keeping one of the constants $\{\alpha, \beta, \gamma, \delta, \mu\}$ non-zero and all others equal to zero in Theorem 3.4 , it follows that the above result of [4] is true even for non-normal complete cone metric space.

Remark 3.8. It has been established in L. J. B. Ciric 2 that a quasi contraction has a unique fixed point in a complete metric space. It follows from the above Remark that the result of 2 is also true for a complete cone metric space even if it is non-normal.

\section{References}

[1] V. Berinde, Itrative approximation of fixed points, Springer Verlag, 2007.

[2] L. J. B. C̈iric,$A$ generalization of Banach contraction princple, Proc. American Mathematical Society 45 (1974), 999-1006. 3.8

[3] L. G. Huang, X. Zhang, Cone metric spaces and fixed point theorems of contractive mappings, J. Math. Anal. Appl. 332 (2007), 1468-1476. 1, 2.1, 2.5, 2.6, 2.8, 2.9, 2.11,3, 3.5

[4] D. Îlic, V. Rakocevic, Quasi-contraction on a cone metric space, Applied Mathematics Letters 22 (2009), 728-731. 2.3 3.6 3. 3.7

[5] Sh. Jain, Sh. Jain and L.B. Jain, Compatibilty and weak compatibility for four self maps in a cone metric space, Bulletin of Mathematical analysis and application 1 (2010), 1-18. 1

[6] Sh. Jain, Sh. Jain and L.B. Jain, Weakly compatibile maps in a cone metric space, Rendiconti Del Seminario Matematica 68 (2010), 115-225. 1

[7] B. V. Kvedaras, A.V. Kibenko and A. I. Perov, On some boundary value problems, Litov. matem. sbornik 5 (1965), 69-84. 1

[8] E. M. Mukhamadiev and V.J. Stetsenko, Fixed point principle in generalized metric space, Izvestija AN Tadzh. SSR, fiz.-mat. i geol.-chem. nauki. 10 (1969), 8-19 [Russian]. 1 ] 
[9] A.I. Perov, The Cauchy problem for systems of ordinary differential equations. In , Approximate methods of solving differential equations, Kiev, Naukova Dumka, 12 (1964), 115-134 [Russian]. 1 ]

[10] A.I. Perov and A.V. Kibenko, An approach to studying boundary value problems, Izvestija AN SSSR, ser. math. 30 (1966), 249-264. [Russian] 1

[11] Sh. Rezapour, R. Hamlbarani, Some notes on the paper "Cone metric spaces and fixed point theorems of contractive mappings", J. Math. Anal. Appl. 345 (2008), 719-724. 1, 2.4, 2.7, 3. 3.5

[12] R. Vasuki, A Fixed Point Theorem for a sequence of Maps satisfying a new contractive type contraction in Menger Space, Math Japonica 35 (1990), 1099-1102.

[13] P.P. Zabrejko, K-metric and K-normed linear spaces Survey Collect. Math. 48 (1997), 825-859. 1 